Gut, 1986, 27, 964-969

Case report

\title{
Neuromuscular and vascular hamartoma of small bowel presenting as inflammatory bowel disease
}

\author{
C E T SMITH, M I FILIPE, AND W J OWEN \\ From the Departments of Clinical Microscopy and Surgery, Guy's Hospital, London
}

SUMMARY We present the case of a rare hamartomatous condition of the small intestine clinically mimicking inflammatory bowel disease. Disorganised fascicles of smooth muscle derived from both musculares mucosae and propria, bundles of non-myelinated nerve fibres with groups of abnormal ganglion cells, and haemangiomatous vessels were present within the submucosa of a long segment of small intestine causing subacute obstruction.

\section{Case report}

A 51 year old Caucasian woman was admitted for explorative laparotomy with a three year history of intermittent lower abdominal pain and occasional episodes of vomiting. A firm, tender, mobile mass, $20 \mathrm{~cm}$ in diameter, was noted in the right abdomen. There was no significant past medical history. Small bowel barium studies showed dilated loops of intestine, with mucosal irregularity and apparent stricture formation (Fig. 1).

At laparotomy a large 'inflammatory' mass involved the jejunum and proximal $80 \mathrm{~cm}$ of ileum. Mesenteric lymphadenopathy was noted. Cryostat sections were carried out and these showed multiple non-caseating granulomata confined to the lymph node and variable degrees of patchy villous atrophy within the mucosa.

A $100 \mathrm{~cm}$ length of small bowel was thus resected for presumed Crohn's disease. Macroscopically this showed variable thickening of the bowel wall with luminal narrowing. Ectatic vessels were visible within the submucosa. The mucosa was oedematous and irregularly ulcerated with areas of classical cobblestone appearance (Fig. 2).

Microscopic examination was undertaken with routine formalin-fixed paraffin-embedded material. Several blocks were taken and sections stained with haematoxylin and eosin and by Elastic van Gieson and Glees and Marsland (neuronal) methods.

Address for correspondence: Dr C E T Smith, Department of Histopathology, Northern General Hospital, Herries Road, Sheffield S5 7AU.

Received for publication 21 November 1985.
Smooth muscle, connective tissue and vascular elements within the submucosa were differentiated by a modified trichrome stain (BVMSB) ${ }^{1}$ and

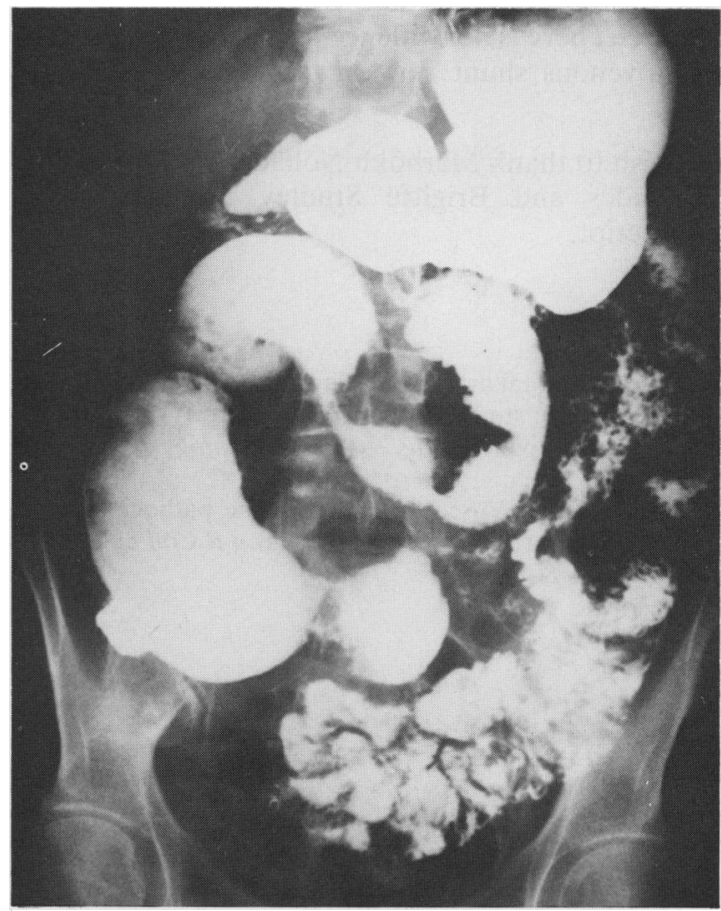

Fig. 1 Radiograph of small bowel barium studies showing widely separated dilated loops of distal jejunum and proximal ileum with mucosal oedema. 


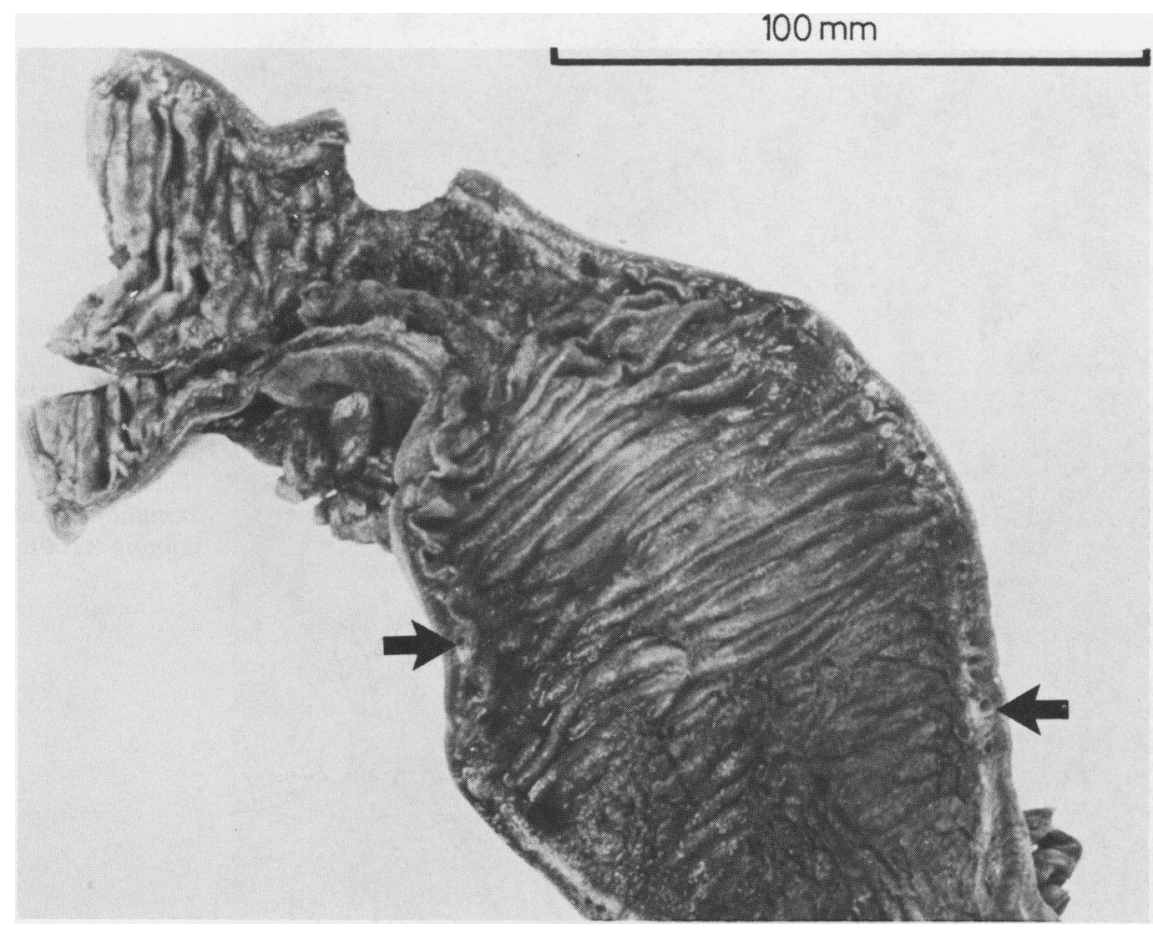

Fig. 2 One end of resection specimen showing an area of dilatation with cobblestone patterns of mucosa and ectatic vessels within the submucosa (arrows).

mucosal argyrophil cells were demonstrated by the Churukion-Schenk method. ${ }^{2}$ Immunohistochemical staining was carried out using antibodies to Factor VIII (endothelium) and S100 protein (neural, melanocyte marker).

Abnormalities were revealed within all layers of the bowel wall. In areas of mucosal ulceration there was marked granulation tissue response, with little fibroblastic proliferation. Arteries and veins were present within the mucosa, some containing thrombus (Fig. 3). Elsewhere the mucosa contained cascades of well formed capillaries, all with prominent endothelial cells (Fig. 4). These vascular channels ascended to the basement membrane of the superficial epithelium and in some areas were related to the presence of fascicles of smooth muscle, arising from the muscularis mucosae, which traversed the lamina propria (Fig. 5). These changes resulted in distortion of mucosal architecture with marked broadening of villi. Multiple foci of pseudopyloric metaplastic change were observed.

Within the submucosa ectatic vascular channels

Fig. 3 A thrombosed artery (left) and vein are present within the mucosa with adjacent dilated capillaries $(H+E \times 64)$.

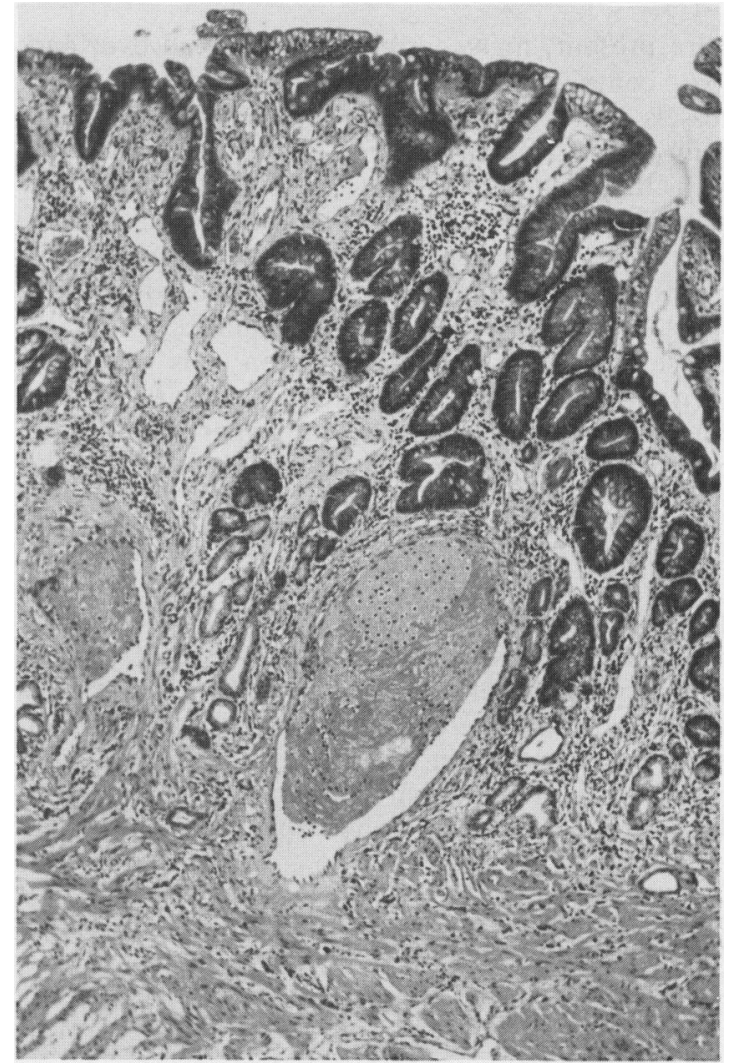




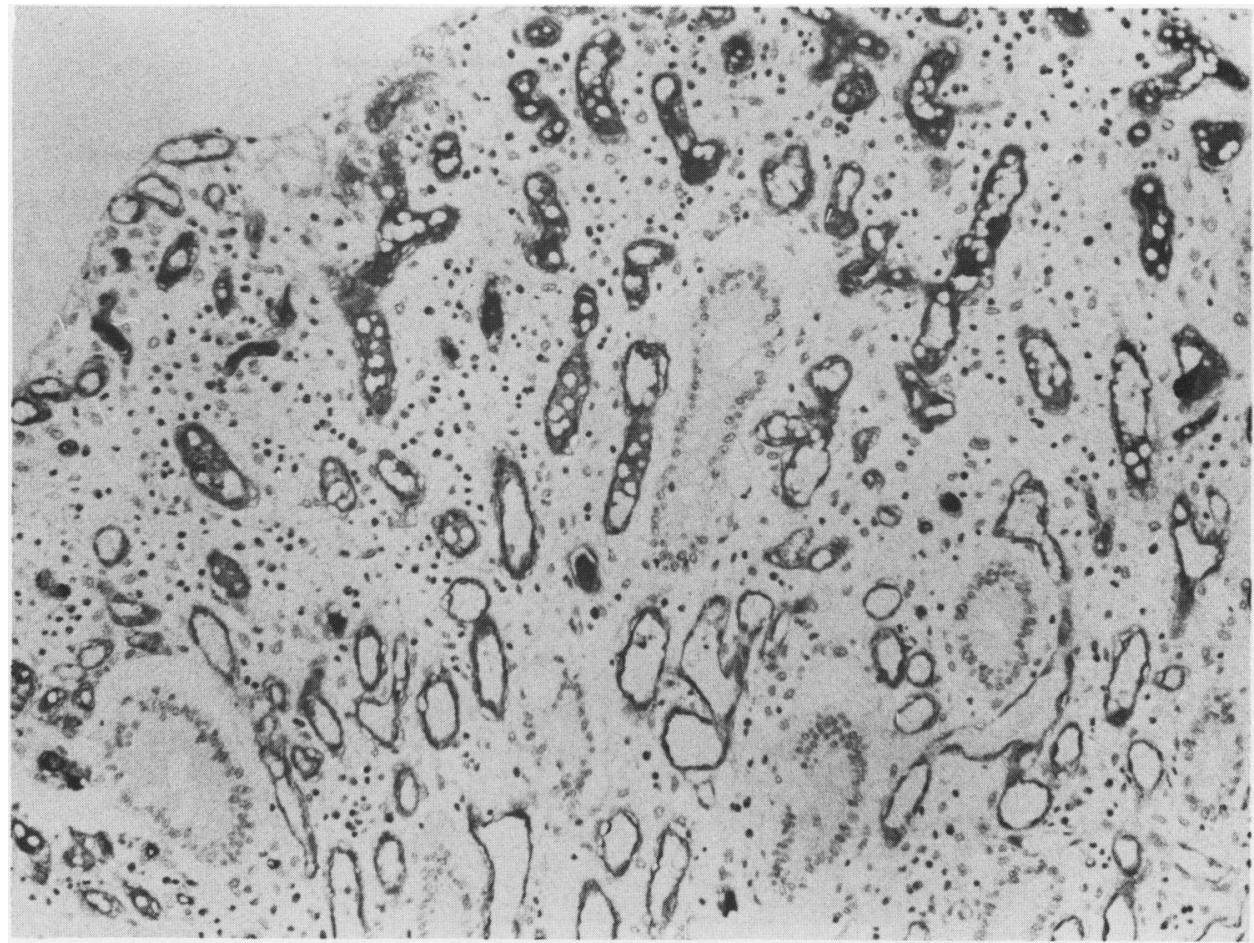

Fig. 4 Numerous vascular spaces within the mucosa (Factor VIII immunoperoxidase staining $\times 160$ ).

were present, many visible to the naked eye, and some contained laminated thrombus (Fig. 6). These vessels possessed walls of variable thickness, many being dilated small arteries. They communicated with both dilated serosal vessels and with the intramucosal capillary proliferations. Large areas of submucosa were replaced by dense, disorganised bands of smooth muscle and collagen with interspersed non-myelinated nerve bundles (Fig. 7). Loose aggregates of giant ganglion cells were also seen (Fig. 8). The muscularis propria showed changes of secondary muscular hypertrophy, with no abnormality of the myenteric plexus.

\section{Discussion}

The findings in this case are those of a nonneoplastic but abnormal arrangement of tissues normally present in the small intestine, and it thus seems most satisfactory to view the changes as of hamartomatous origin. Neuromuscular and vascular hamartoma of the small bowel is extremely rare. Two cases were described by Fernando and

Fig. 5 Smooth muscle fibres vertically arranged within the abnormal mucosa $(H+E \times 160)$.

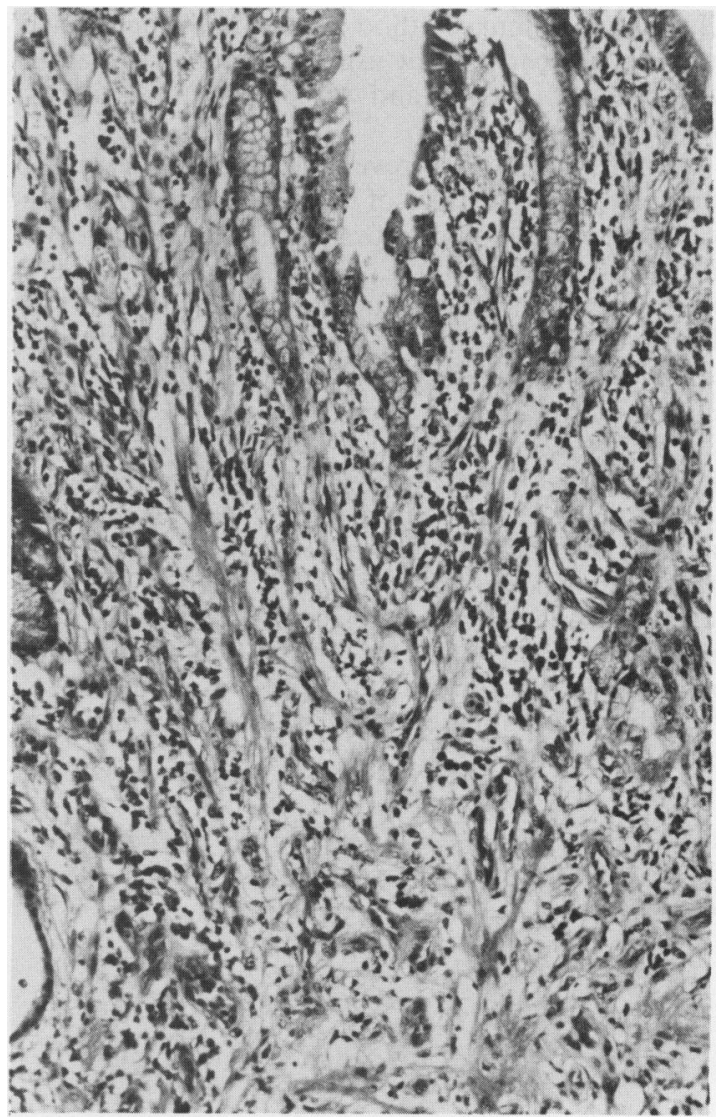




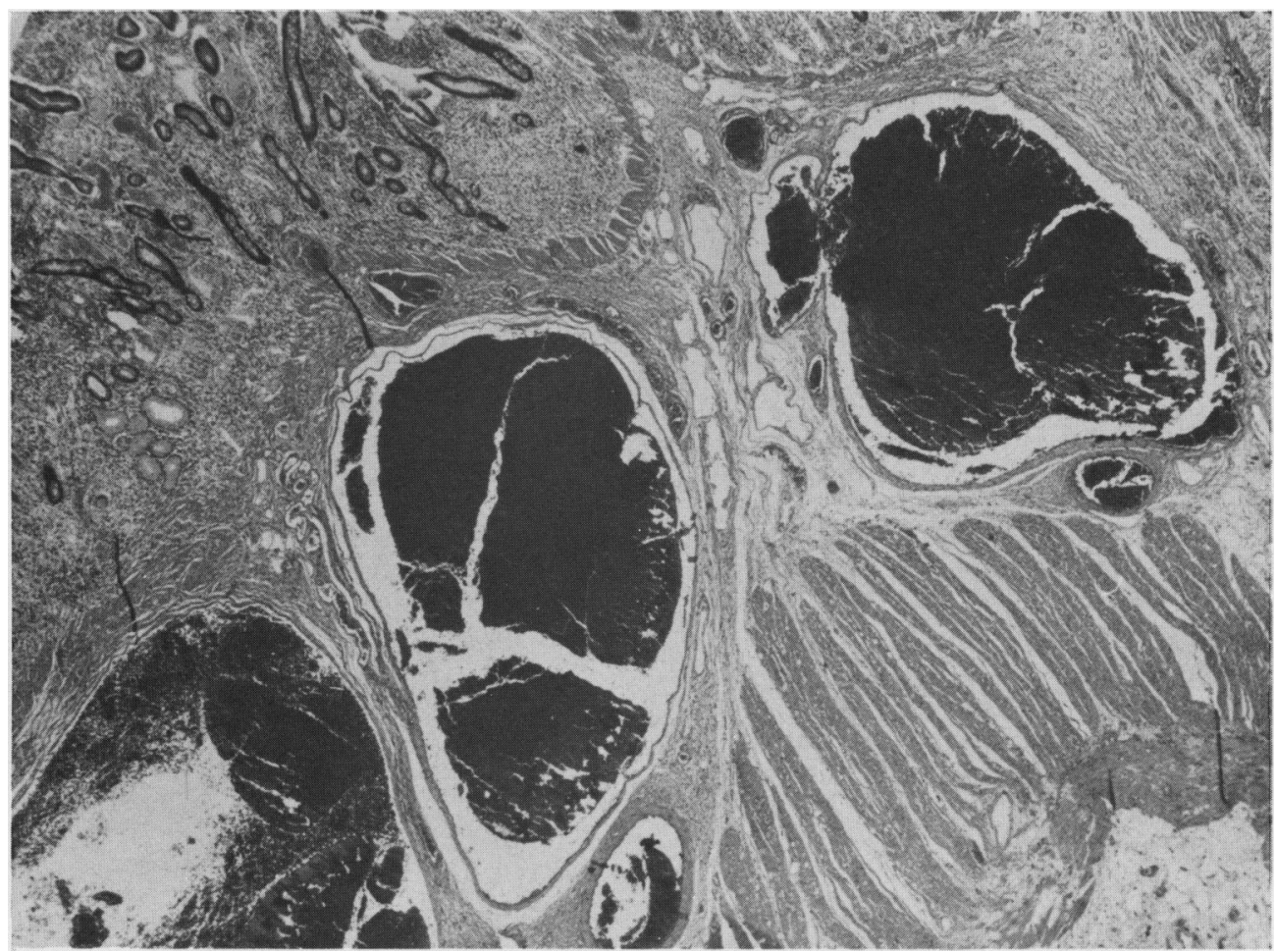

Fig. 6 Dilated vessels within the submucosa. The mucosa contains foci of pseudopyloric metaplasia $(H+E \times 25)$.

McGovern in $1982^{3}$ and these remain, to our knowledge, the only two reported cases. As in our case, both of these showed stenotic segments of bowel associated with mucosal ulceration and aberrant fascicles of muscle derived from the muscularis muscosae entering the submucosa, together with bundles of non-myelinated nerve fibres, scattered ganglion cells and abnormal blood vessels. In both cases the segments of affected bowel were shorter than $5 \mathrm{~cm}$, one case having multiple such foci. In our patient the changes were strikingly diffuse throughout a much greater length of bowel.

No abnormalities of neuroendocrine cells were shown in either our case or the two previously described. In the former cases no abnormalities were noted within regional lymph nodes. Our case displayed mesenteric lymphadenopathy with noncaseating granulomata. There were no mucosal stigmata of Crohn's disease or tuberculosis and further investigation in search of acid fast bacilli proved negative. There were no clinical or radiological features of sarcoid and the resection specimen showed none of the changes of Whipple's disease. The lymphadenopathy thus appears to be a reflection of the degree and extent of mucosal ulceration. At six month follow up the patient is entirely well.

Hamartomatous lesions of the small bowel in which neural elements participate are classified by Morson and Dawson ${ }^{4}$ as part of the spectrum of neuromatous hyperplasia. Although the presence of abnormal neuronal elements was striking in our case, other changes, notably those involving vessels, seemed equally florid. This lesion appears unrelated to ganglioneuromas, paragangliomas, and glomus tumours, which are all more discreetly localised. Neuronal colonic dysplasia is similarly characterised by the widespread increase in numbers of abnormal ganglion cells and nerves, but there are no accompanying abnormalities of smooth muscle or vessels. $^{5}$ The same is true of plexiform neurofibromatosis and our patient showed no manifestation of a more widespread or multisystem disease. This lesion thus appears to be an isolated and distinct entity.

We wish to thank $\operatorname{Dr} M \quad R$ Salari, Lewisham Hospital, for access to the radiological studies and Mr S. Buk for technical and histochemical assistance. 


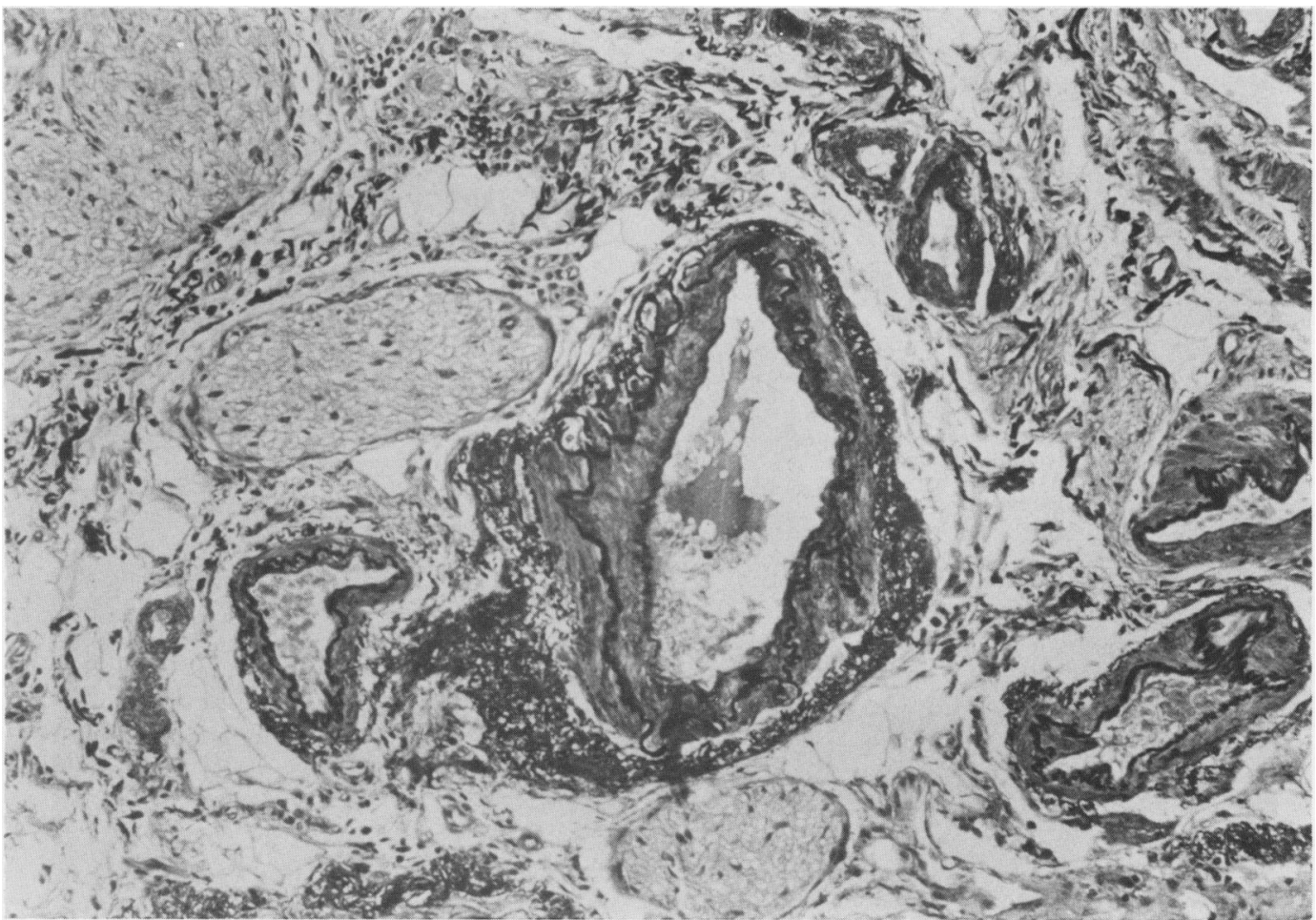

Fig. 7 Non-myelinated nerve bundles within the submucosa, in association with arteries (BVMSB $\times 160)$.

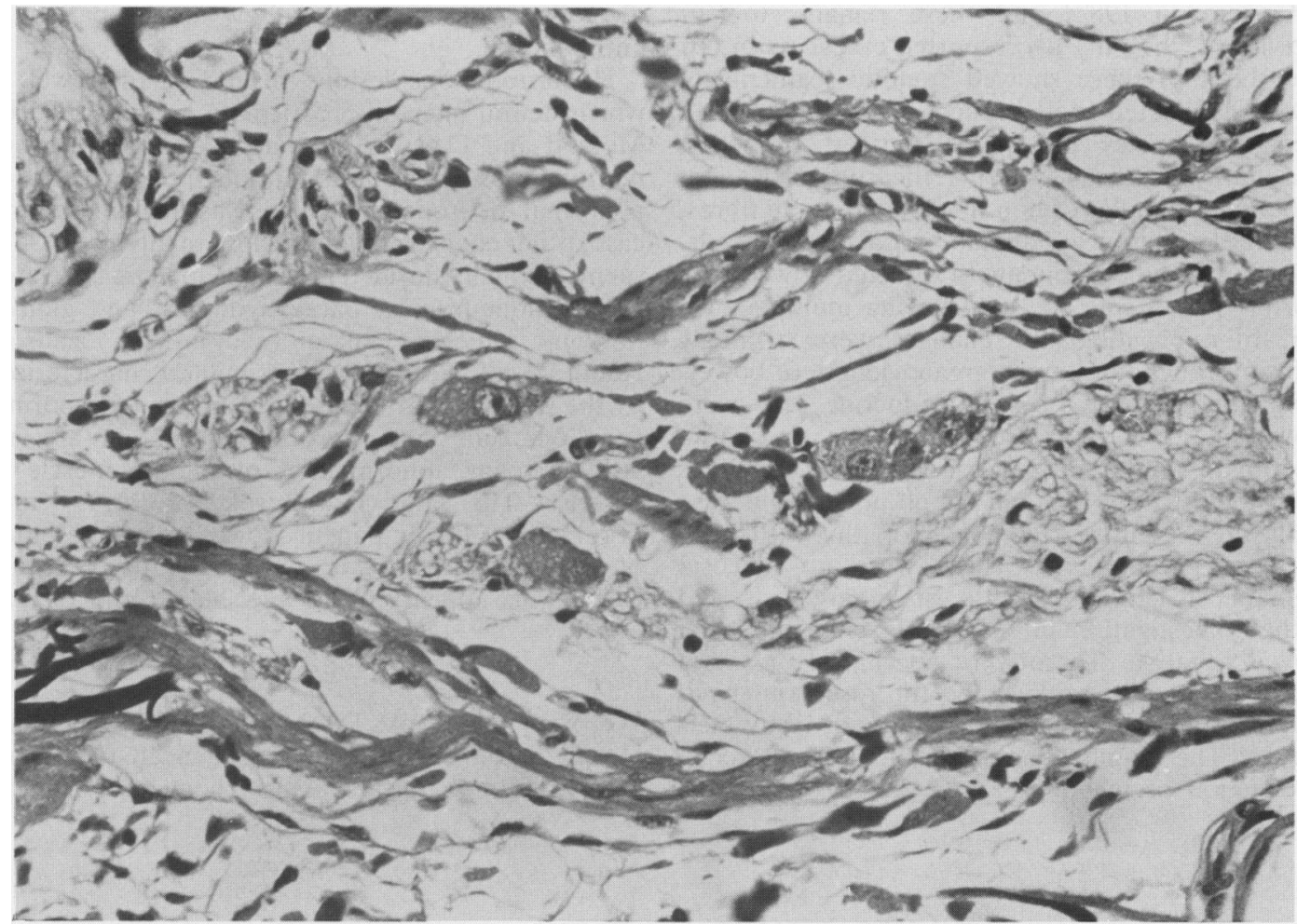

Fig. 8 Giant ganglion cells with loosely arranged nerve fibres and smooth muscle fibres within the submucosa $(H+E \times 320)$. 


\section{References}

1 Buk SJA. Simultaneous demonstration of connective tissue, elastica and fibrin by a combined Verhoeffs Elastic - Martius-Scarlet-Blue trichrome stain. Stain Technol 1984: 59: 1-5.

2 Churukian CJ, Sckenk EA. A modification of Pascual's Argyrophil Method. J Histotechnol 1979; 2: 102-3.
3 Fernando SSE, McGovern MJ. Nuromuscular and vascular hamartoma of small bowel. Gut 1982; 23: 1008-12.

4 Morson BC, Dawson IMP. Gastro-intestinal pathology. Oxford: Blackwell, 1979: 425-6.

5 Meier-Ruge W. Hirschprung's disease: its aetiology, pathogenesis and differential diagnosis. Berlin: Springer-Verlag, 1974: 163-5. 